\title{
Brustzentrum Halle/Saale: Gut gerüstet für die Zukunft
}

\author{
Eva J. Kantelhardt ${ }^{\mathrm{a}}$ Regina Grosse ${ }^{\mathrm{a}} \quad$ Martina Vetter $^{\mathrm{a}} \quad$ Anke Heinig $^{\mathrm{b}}$ Hans-Jürgen Holzhausen ${ }^{\mathrm{c}}$ \\ Thomas Kegel $^{d} \quad$ Andrea Diestelhorst ${ }^{e}$ Traute Mende ${ }^{f} \quad$ Hans-Georg Strauss $^{a}$ \\ Christoph Thomssen ${ }^{a}$
}

aUniversitätsklinik für Gynäkologie, bUniversitätsklinik für Radiologie, ' Institut für Pathologie, dUniversitätsklinik für Innere Medizin,

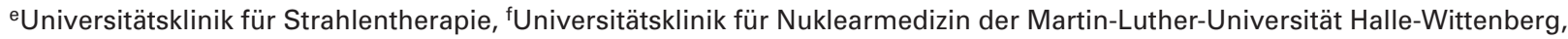
Deutschland

\section{Erstes zertifiziertes Brustzentrum in den neuen Bundes- ländern}

Das Brustzentrum am Universitätsklinikum der MartinLuther-Universität Halle-Wittenberg wurde im Januar 2004 als erstes Brustzentrum in den neuen Bundesländern nach den Kriterien der DKG/DGS und ISO 9001 zertifiziert. Neben der Klinik und Poliklinik für Gynäkologie und der Klinik für Diagnostische Radiologie sind mit den Kliniken und Instituten für Pathologie, Nuklearmedizin, Strahlentherapie und Internistische Onkologie als Teile des Universitätsklinikums alle Behandlungspartner unter einem Dach. Eine Psychologin arbeitet mit $100 \%$ in der Klinik für Gynäkologie. Das Forschungslabor der Gynäkologie mit zwei Biologen und zwei MTAs gewährleistet die Umsetzung der translationalen Forschung auf hohem Niveau. Im Studiensekretariat sind eine StudyNurse und eine Dokumentarin tätig, sie betreuen klinische Studien und führen die Datenbank. Die Sachsen-Anhaltische Krebsgesellschaft ist als Vertreter der Patientinnen-Gruppen integriert.

\section{Betreuung der Patientinnen}

\section{Erstkontakt}

Der Erstkontakt der Patientin mit dem Brustzentrum Halle erfolgt per Überweisung der niedergelassenen Kollegen an die Senologische Ambulanz der Klinik und Poliklinik für Gynäkologie. Diese Polikliniken sind schon sehr lange eine gut etablierte Schnittstelle zwischen den niedergelassenen Kollegen und dem Universitätsklinikum Halle. Die Eingangsdiagnostik erfolgt gemeinsam mit den Kollegen der Diagnostischen Radiologie (im gleichen Gebäude ein Stockwerk höher) - falls notwendig einschließlich der minimal-invasiven, bildgebend gesteuerten Stanzbiopsie zur histologischen Sicherung der Di- agnose. Die Befunde werden im interdisziplinären Konsil unter Beteiligung von Gynäkologen, Radiodiagnostikern, Pathologen und Strahlentherapeuten angesehen und besprochen. Das weitere Vorgehen wird festgelegt und unmittelbar dokumentiert.

\section{Diagnostische Schritte}

Notwendige und ergänzende diagnostische Schritte werden eingeleitet. Die Klinik für Diagnostische Radiologie bietet neben den kompletten diagnostischen Möglichkeiten auch die bildgestützte minimal-invasive histologischen Sicherung auf der Basis von Mammographie, Mammasonographie und Magnetresonanztomographie (MRT) mittels Hochgeschwindigkeits- und Vakuumbiopsien an. Benigne Veränderungen können im Einzelfall durch die Vakuumbiopsie vollständig entfernt werden. Bei Indikation zu einer offenen Operation wird bereits jetzt, soweit möglich, das lokoregionäre Therapiekonzept diskutiert und festgelegt. Bei Planung eines brusterhaltenden Vorgehens wird bei fast allen Patientinnen mit histologisch gesichertem Mammakarzinom eine präoperative MRTDiagnostik durchgeführt. Bei Zusatzbefunden wird zur gezielten Abklärung eine zusätzliche, gegebenenfalls MRT-gestützte Stanzbiopsie entnommen. Somit erfolgt präoperativ die «Kartierung» der betroffenen Brust und der Ausschluss von kontralateralen Zweitherden. Eine Auswertung des aufwendigen Vorgehens ist derzeit in Arbeit.

\section{Adjuvante Therapie}

Nach Vorliegen des histologischen Befundes und unter Berücksichtigung der bildgebenden Diagnostik wird jede Patientin mindestens ein zweites Mal in der interdisziplinären Konferenz besprochen, um adjuvante Therapiemaßnahmen und die Art der Nachsorge interdisziplinär zu diskutieren und festzulegen. Brusterhaltende Therapie oder Mastektomie, Sentinel-Lypmhknoten-Exzision oder Axilladissektion, Bestrah-

\begin{tabular}{ll}
\hline KARGER & ( ) 2007 S. Karger GmbH, Freiburg \\
Fax +49 7614520714 & Accessible online at: \\
$\begin{array}{l}\text { E-mail Information@Karger.de } \\
\text { www.karger.com }\end{array}$ & www.karger.com/brc
\end{tabular}


lung der Brust, Brustwand oder Lymphknotenstationen, einschließlich der plastischen Rekonstruktion mit myokutanen Lappenplastiken oder alternativ allogenem Material werden besprochen. Die Empfehlungen zur Systemtherapie halten sich dabei an die aktuellen Empfehlungen der AGO und der DKG. Vorzugsweise werden die Patientinnen in multizentrische klinische Studien eingebracht. Die Therapien werden, wenn immer möglich, tagesklinsich oder ambulant appliziert. Wir bieten neben den bekannten großen adjuvanten Studien (NNBC-3 Europe, GAIN, GeparQuinto, ICE, PACT) auch spezielle Therapien für bestimmte Indikationen als Phase-II-, und -III-Studien an. Eine enge Zusammenarbeit erfolgt mit der AGO, GBG, EORTC, BIG, NOGGO und WSG.

\section{Stationäre Betreuung}

Auf der Station betreuen neben den Ärzten in Weiterbildung insbesondere die drei senologisch-operativ tätigen Fachärzte, ein Arzt mit der Zusatzweiterbildung «Medikamentöse Tumortherapie» und mehrere onkologisch weitergebildete Schwestern die Patientinnen mit Mammakarzinom. Die in der Klinik integrierte psychoonkologische Betreuung, die auch ambulanten Patientinnen zur Verfügung steht, bietet neben der Krisenintervention auch Paar- und Familienbetreuung, geleitete Imagination und Entspannungsverfahren an. Kunsttherapie sowie Sport- und Ernährungsberatung werden zukunftsorientiert als Komplementärtherapien angeboten. Ein hiesiges Sanitätshaus gewährleistet die Betreuung mit Hilfsmitteln auf der Station.

\section{Tagesklinik und Nachsorge}

Nach der Operation erfolgt die weitere Betreuung der Patientinnen bei erforderlicher Chemotherapie in der Tagesklinik. Zwei Assistenzärzte, ein Oberarzt und zwei Schwestern sind dort eingesetzt und können sich ausschließlich der ambulanten/teilstationären Betreuung widmen. Besonders für Patientinnen mit metastasiertem Mammakarzinom ist diese Kontinuität wichtig. Durch enge Zusammenarbeit mit unserem Studiensekretariat können neue Substanzen wie spezifische Antikörper oder «small molecules» für diese Patientinnen angeboten werden.

Die Nachsorge der Patientinnen in der senologischen Sprechstunde der Hochschulambulanz erfolgt nach Überweisung durch die niedergelassenen Kollegen, welche die übliche gynäkologische Vorsorge durchführen. Auch im DMP-Programm werden hier Patientinnen betreut. Unsere Nachsorge wird entsprechend der Leitlinien der DKG durchgeführt.

\section{Translationale Forschung}

Um translationale Forschung zu ermöglichen, besteht eine enge Zusammenarbeit zwischen unserem Brustzentrum und dem Forschungslabor der Klinik. Von jeder Patientin wird im Rahmen prospektiver Studien nach Möglichkeit zusätzlich zur
Probe für Histologie und Diagnostik sowohl aus der Stanzbiopsie als auch aus dem operativ entfernten Tumor Gewebe eingefroren. Die Asservierung wurde in den letzten 3 Jahren optimiert und wird von speziell eingearbeitetem Personal durchgeführt. Versuchsreihen zu Lagerungszeiten, Lagerungstemperaturen und Einfluss der Tumorheterogenität auf Entnahmestellen wurden durchgeführt. Den Patientinnen mit G2Tumoren und negativem Nodalstatus bieten wir die Bestimmung von uPA/PAI-I als zusätzlichen empfohlenen Prognosemarker an. Im Rahmen der NNBC-3 Europe Studie kann so vielen Patientinnen eine Chemotherapie erspart werden. Die biochemische Bestimmung von uPA und PAI-I bieten wir deutschlandweit für alle Kliniken an, die schockgefrorene Gewebeproben auf Trockeneis einsenden. Die Vertiefung der Kooperation zwischen Klinik und Labor im Bereich der Forschung erfolgt durch eine Rotation der Kliniksärzte ins Labor. Bearbeitete Themen sind z. B. Übereinstimmung von Stanzbiopsie und Tumor für HER-2 und uPA, PAI-1. Neue Fragestellungen für bestimmte Patientengruppen werden z. B. im Rahmen von Doktorarbeiten bearbeitet.

\section{Ausblick}

Die Strukturierung der Arbeit in der oben beschriebenen Weise hat intern für die Mitarbeiter und extern für die Einweiser einen erheblichen Zugewinn an Transparenz erbracht. Die steigenden Patientinnenzahlen belegen die hohe Akzeptanz, die das Brustzentrum bei den Kooperationspartnern genießt.

Das Re-Zertifizierungsverfahren nach zwei Zwischenaudits hat das Brustzentrum Halle problemlos bestanden. Anhand der Zahlen aus den letzten 5 Jahren können Entwicklungen und Trends nicht nur durch die erhobenen Daten, sondern auch durch errechnete Parameter aus Tumorbiologie, Therapiekonzepten und Nachbeobachtung erkannt werden. Im nationalen «benchmarking» (Westdeutsches Brustzentrum, Bundesgeschäftsstelle Qualitätssicherung gGmbH) kann die Leistung unserer Klinik gut eingestuft werden.

Für die Zukunft sind weitere Herausforderungen abzusehen! Wie wird die Einbindung des Mammographie-Screenings in die bestehende Arbeit gelingen? Welche neuen Prognosefaktoren werden sich im Zeitalter der Gene-Arrays durchsetzen und Therapieentscheidungen beeinflussen? Gibt es neue Substanzen? Wie sollen sich die DMP-Programme als parallele Struktur zu den Anforderungen an die Brustzentren weiterentwickeln? Wird es konsensfähige Vorschläge geben, sich auf einheitliche Dokumentationsvariablen zu einigen - wird es weniger Mehrfachdokumentationen geben? Kann eine enge Zusammenarbeit zwischen stationären und ambulanten Einrichtungen im Rahmen der Nachsorge erfolgen, so dass eine kontinuierliche Dokumentation möglich ist? Gibt es Hoffnung auf ein einheitliches Krebsregister mit adäquater personeller Versorgung? Wie kann der Mehraufwand eines Brust- 
zentrums in den DRGs abgebildet werden ohne die Behandlung für Krankenkassen unattraktiv werden zu lassen?

Wir sind gespannt auf die Entwicklung in den nächsten 3 Jahren - gerade auch in den neuen Ländern!

\section{Weiterführende Literatur}

EUSOMA: The requirements of a specialist breast unit. Eur J Cancer 2000;36: 2288-93.

Deutsche Krebsgesellschaft: Zertifizierung - Qualitätsstandards für Brustzentren. http://www.krebsgesellschaft.de/wub_zertifizierung,10096.html.
Cataliotti L, Costa A, Daly PA, Fallowfield L, Freilich G, Holmberg L, Piccart M, van de Velde CJ, Veronesi U: Florence statement on breast cancer, 1998 forging the way ahead for more research on and better care in breast cancer. Eur J Cancer 1999;35:14-5.

Blamey RW, Cataliotti L: EUSOMA accreditation of breast units. Eur J Cancer 2006;42:1331-7.

Disease Management Programm: Vertrag zwischen der AOK Rheinland, dem Landesverband der Betriebskrankenkassen NRW, der Innungskrankenkasse Nordrhein der Landwirtschaftlichen Krankenkasse NRW, dem VdAK/AEV Landesvertretung NRW, der Bundesknappschaft und der Kassenärztlichen Vereinigung Nordrhein über ein strukturiertes Behandlungsprogramm (DMP) zur Verbesserung der Versorgungssituation von Brustkrebspatientinnen. http://www.kvno.de/ mitglieder/vertraeg/dmp_mama/index.html.

Einführung eines bundesweiten Mammographie-Screening-Programmes. Beilage zum Deutschen Ärzteblatt, Heft 4 vom 23.1.2004 Ausgabe A. 